\title{
Successful Detection of a Preferred Frame Using Eclipses of Jupiter's Moon Io
}

\author{
Stephan J. G. Gift \\ Department of Electrical and Computer Engineering, Faculty of Engineering, The University of the West Indies, St. Augustine, \\ Trinidad and Tobago \\ Email: Stephan.Gift@sta.uwi.edu
}

How to cite this paper: Gift, S.J.G. (2022) Successful Detection of a Preferred Frame Using Eclipses of Jupiter's Moon Io. Journal of Applied Mathematics and Physics, 10, 322-332.

https://doi.org/10.4236/jamp.2022.102025

Received: July 23, 2021

Accepted: February 14, 2022

Published: February 17, 2022

Copyright (c) 2022 by author(s) and Scientific Research Publishing Inc. This work is licensed under the Creative Commons Attribution International License (CC BY 4.0).

http://creativecommons.org/licenses/by/4.0/

(c) (i) Open Access

\begin{abstract}
In this paper tests by Maxwell and Gift that search for a preferred frame or ether arising from movement through that frame using Jupiter's moon Io are reviewed and discussed. Unlike the Michelson-Morley second-order experiment which unsuccessfully attempted to detect the orbital motion of the Earth relative to the ether, these tests are both first-order and therefore are unaffected by the second-order effects of length contraction and clock retardation. The test by Maxwell utilizes the delay in the eclipse of Io as the Earth orbits the Sun in an attempt to detect ether drift resulting from the galactic movement of the Sun. This test requires a 6-year duration for its full execution and was never performed because of practical difficulties. The test first presented by Gift can be conducted over a few days and employs the observed variation of the period of Io as the Earth moves toward or away from Jupiter. The result is a positive detection of ether drift arising from the orbital motion of the Earth. The detected ether drift is evidence of a preferred frame which we argue corresponds to the solar system barycentric or sun-centered inertial (SCI) frame.
\end{abstract}

\section{Keywords}

Ether Drift, Maxwell, Roemer, Jupiter, Io, Luminiferous Ether, Preferred Frame, Sun-Centered Inertial Frame

\section{Introduction}

The luminiferous ether, a medium permeating all space and considered to be the carrier of light waves, was believed to exist based on evidence accumulated over many years [1] [2]. This belief was reinforced by the discovery of electromagnetic waves in 1879, in which Maxwell correctly predicted travel through this medium at speed $c=1 / \sqrt{\varepsilon_{0} \mu_{o}}$ where the electric permittivity $\varepsilon_{0}$ and the mag- 
netic permeability $\mu_{o}$ are defined and measurable characteristics of that medium. Michelson and Morley, in their famous experiment of 1887 [3], searched for direct evidence of the ether. This experiment utilized interfering beams of light that travelled along orthogonal paths on a movable apparatus. The system was designed to reveal the speed of the Earth's orbital motion through the hypothesized ether based on changes in light speed resulting from movement with or against the associated ether wind. The predicted fringe shift $\delta$ is given by [4]

$$
\delta=\frac{2(v / c)^{2}}{\lambda / l}
$$

where $v$ is the orbital speed of the Earth through the ether, $l$ is the arm length of the interferometer and $\lambda$ is the wavelength of the light. The observed fringe shift was significantly less than that expected as a result of the orbiting Earth and this essentially null result was interpreted as an indication of light speed constancy in all directions on the surface of the Earth and hence the nonexistence of an ether. This idea of light speed constancy later formed the foundation of the special theory of relativity which directly prohibits an ether (or preferred frame).

A well-known characteristic of Michelson-Morley type experiments (in a vacuum) that is evident in Equation (1) is they are second-order, i.e. the effect to be observed is proportional to $\left(\mathrm{V}^{2} / \mathrm{c}^{2}\right)$, since the light travels out-and-back resulting in the cancellation of first-order effects. Thus, if the (one-way) distance travelled by the light is $I$ and the initial direction of light travel is coincident with the direction of movement of the Earth, then the time to travel this path and return to the start is given by

$$
t=\frac{l}{c-v}+\frac{l}{c+v}=\frac{2 l c}{c^{2}-v^{2}} \simeq \frac{2 l}{c}\left(1+\frac{v^{2}}{c^{2}}\right)
$$

The difference $\Delta t$ between this time and the time in the absence of motion is given by

$$
\Delta t=\frac{2 l}{c}\left(1+\frac{v^{2}}{c^{2}}\right)-\frac{2 l}{c}=\frac{2 l}{c} \frac{v^{2}}{c^{2}}
$$

As can be seen from (3), there are no first-order effects in this out-and-back result with only second-order effects remaining. Such experiments are therefore directly affected by the second-order effects of time dilation [5] and length contraction [6] thereby rendering them unreliable for the detection of ether drift. In fact, Gift [7] and Levy [8] have shown that in an ether theory, two-way light speed measurement will always give the light speed in the underlying light-carrying ether medium, a finding that is independent of the movement of the platform on which the measurement is made. This amazing result means that in the framework of an ether theory, two-way light speed measurement will always give a constant value, even though the one-way light speed is not! This two-way light speed constancy has been experimentally confirmed [9]. It gives the illusion of one-way light speed constancy [7] and therefore the perception of the nonexistence of the ether. 
Towards the detection of ether drift, Maxwell had long ago argued that first-order experiments are superior for this action [10]. However, in a paper in Nature marking the centenary of the introduction of the theory of relativity, Stachel stated [11] "all attempts to detect the translational motion of the Earth through the ether by means of optical, electrical or magnetic effects consistently failed. Lorentz succeeded in explaining why: according to his theory, no such effect should be detectable by any experiment sensitive to first order in $(v / c)$ where $V$ is the speed of the moving object through the ether and $c$ is the speed of light in that medium." This statement about Lorentz's theory is misleading.

What this theory in fact established is that in experiments utilizing closed light paths, no first-order effects are detectable since in such experiments first-order terms cancel as discussed earlier [12]. Lorentz, who was aware of the failure of the Michelson-Morley experiment, stated [13], "The problem of determining the influence exerted on electric and optical phenomena by a translation, such as all systems have in virtue of the Earth's annual motion, admits of a comparatively simple solution, so long as only those terms need be taken into account, which are proportional to the first power of the ratio between the velocity of translation $v$ and the velocity of light $c$. Cases in which quantities of the second order, i.e. of the order $v^{2} / c^{2}$, may be perceptible, present more difficulties." Contrary to Stachel therefore, Lorentz actually recommended first-order experiments in attempts to detect movement through the ether. Maxwell indeed suggested a first-order method to detect the movement of the solar system through the ether using the delay in the eclipse time of Jupiter's satellite Io first observed by Roemer [14]. It required six years for its execution and was not pursued at the time because of inadequate equipment sensitivity.

In this paper we review and discuss two first-order tests for detecting ether drift. The first is the test suggested by Maxwell [4] [15] that seeks to detect movement of the Sun through the ether by utilizing the eclipses of Jupiter's satellite Io. A modification is suggested that shortens the duration of the test from 6 years to 6 months. The second is a first-order test first discussed by Gift [16] which utilizes variations in the period of Jupiter's satellite Io to detect the orbital movement of the Earth relative to the ether, the movement that Michelson and Morley tried unsuccessfully to detect. Maxwell's approach requires 6 years and was never conducted while Gift's approach requires only a few days and gives a confirmed positive result.

\section{Detection of the Galactic Motion of the Sun Using Maxwell's Experiment}

In an 1875 entry in the Encyclopedia Britannica in support of the ether and its existence [10], Maxwell wrote "If it were possible to determine the velocity of light by observing the time it takes to travel between one station and another, we might by comparing the observed velocities in opposite directions, determine the velocity of the ether with respect to these terrestrial stations." Recognizing 
the difficulty of this exercise since it involved second-order methods, Maxwell later suggested a first-order experiment for detecting movement of the Sun through the ether that involves observing the eclipses of Jupiter's moon Io [4] [15].

Consider Figure 1 where the Earth and planet Jupiter orbit the Sun which is moving with velocity $v$ in the direction shown. Since Jupiter has a period of 12 (terrestrial) years, in half a year as Earth moves from $A$ to $B$, Jupiter moves only a short distance from $A^{\prime}$ in its orbit. Therefore, by timing the delay of consecutive eclipses of Io with Earth first at $A$ and moving later to $B$, then the time $t_{1}$ for light to traverse the path from $A$ to $B$ a distance equal to the diameter $l$ of the Earth's orbit can be determined. In this case the solar system would be moving towards the occulting light at speed $v$ and hence light speed would be $c+v$. This time is given by

$$
t_{1}=\frac{l}{c+v}
$$

Six years later when Jupiter is at point $B^{\prime}$ in its orbit, the measurement of eclipse delay is repeated with Earth initially at $B$ and later at $A$. This would again give the time $t_{2}$ for the light to travel from $B$ to $A$ at speed $c-v$ since the solar system would be moving away from the occulting light at speed $v$ relative to the ether. This time is given by

$$
t_{2}=\frac{l}{c-v}
$$

The difference between these times $\Delta t=t_{2}-t_{1}$ is given by

$$
\Delta t=\frac{l}{c-v}-\frac{l}{c+v}=\frac{l(c+v-c+v)}{c^{2}-v^{2}}=\frac{2 l v}{c^{2}-v^{2}}
$$

This reduces to

$$
\Delta t \simeq 2 t_{o} \frac{v}{c}, v \ll c
$$

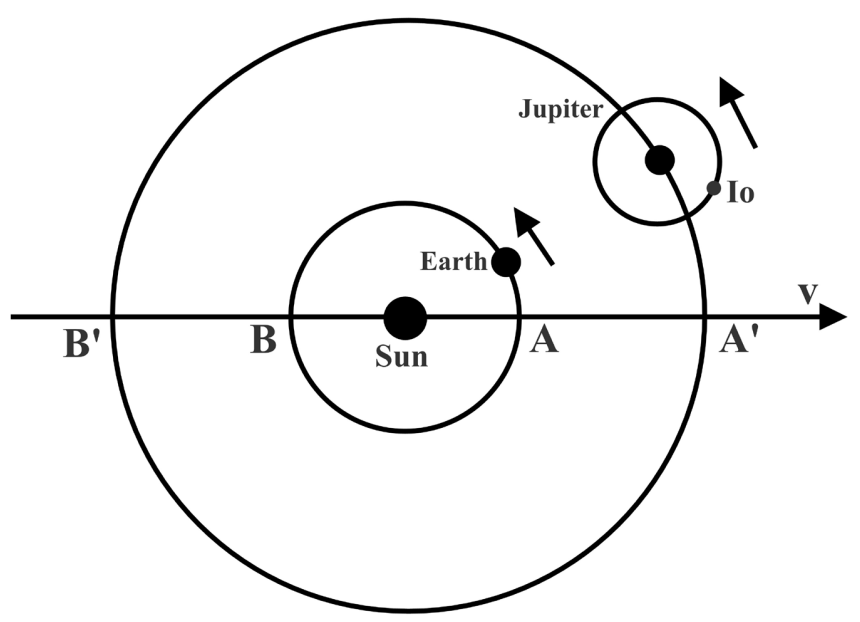

Figure 1. Motion of Sun-Earth-Jupiter system through the ether. 
where $t_{o}=l / c$. This result (7) is first-order and therefore unaffected by secondorder effects of clock retardation and length contraction as is the second-order result given in Equation (1) of the Michelson-Morley experiment although both experiments seek to detect a velocity $v$. Using the orbital speed of the Sun as $v=2.3 \times 10^{5} \mathrm{~m} / \mathrm{s}$ and $t_{o}=1000 \mathrm{~s}$ (since the time for light to travel from the Sun to the Earth is 500s), then the predicted time change is given by

$$
\Delta t \simeq 2 \times 1000 \times \frac{2.3 \times 10^{5}}{2.998 \times 10^{8}}=1.5 \mathrm{~s}
$$

This experiment was never performed because of the inaccuracies associated with uncontrolled variables such as variations in the orbits of Jupiter and the Earth over two 6-month periods six years apart.

A modification of Maxwell's experiment that reduces the time over which the measurements must be made from 6 years to 6 months is to use one of the eclipse-delay measurements say $t_{1}$ and compare it with the time $t_{o}$ for light to travel across the diameter of the Earth's orbit at speed $c$ given by $t_{o}=l / c$. This latter time has been independently determined and is known. The difference between these times $\Delta t_{1}^{\prime}=t_{o}-t_{1}$ is the time change caused by motion of the Sun and is given by

$$
\Delta t_{1}^{\prime}=\frac{l}{c}-\frac{l}{c+v}=\frac{l(c+v-c)}{c(c+v)}=\frac{l v}{c(c+v)}
$$

This reduces to

$$
\Delta t_{1}^{\prime} \simeq t_{o} \frac{v}{C}, v \ll c
$$

A time difference $\Delta t_{2}^{\prime}=t_{2}-t_{o}$ using the eclipse-delay measurement $t_{2}$ can also be computed and is given by

$$
\Delta t_{2}^{\prime}=\frac{l}{c-v}-\frac{l}{c}=\frac{l(c-c+v)}{c(c-v)}=\frac{l v}{c(c-v)}
$$

This reduces to

$$
\Delta t_{2}^{\prime} \simeq t_{o} \frac{v}{c}, v \ll c
$$

which is the same as (10). Thus, either delay $t_{1}$ or $t_{2}$ can be used but both are not required thereby eliminating the 6-year wait. While the predicted time delay in (10) and (12) is half of the value predicted in (7) in the original experiment, the time $t_{o}$ is simply twice the time for light to travel from the Sun to the Earth at speed $c$, a value that has been independently determined. Therefore, there is no need to spend 6 years conducting the test; it can be completed in the six-month period required for the measurement of $t_{1}$ or $t_{2}$. Using the orbital speed of the Sun as $v=2.3 \times 10^{5} \mathrm{~m} / \mathrm{s}$ and $t_{o}=1000 \mathrm{~s}$, the expected time change arising from motion of the Sun is

$$
\Delta t^{\prime} \simeq 1000 \times \frac{2.3 \times 10^{5}}{2.998 \times 10^{8}}=0.77 \mathrm{~s}
$$


This can easily be measured using modern atomic clocks which are both accurate and stable. Therefore, we suggest that Maxwell's first-order experiment in this modified form be repeated in order to detect ether drift associated with the galactic movement of the Sun since it can be conducted over a period of six months instead of six years.

\section{Detection of the Orbital Motion of the Earth Using Roemer's Observation}

A method which like Maxwell's experiment utilizes the eclipses of Io but represents a major improvement of that test since it can be conducted in a few days, is one that is based on an important observation by Roemer. In 1676 Roemer observed that Io undergoes a regular variation in its period of revolution about Jupiter as the Earth orbits the Sun. As the Earth approaches Jupiter, the satellite's period decreases while as the Earth recedes from Jupiter, the satellite's period increases. Gift used this observation to detect ether drift [16] arising from the Earth's orbital motion, precisely the motion that the Michelson-Morley experiment tried unsuccessfully to detect. Now as seen from the Earth, Io is periodically eclipsed by Jupiter thereby resulting in the emission of what can be viewed as "pulses of darkness" that travel to Earth at speed $C$ relative to the ether as Io orbits Jupiter. These pulses are represented in Figure 2.

The distance between successive pulses is fixed at a value $\lambda_{o}$ and is related to the period $T_{o}$ by the equation

$$
T_{o}=\frac{\lambda_{o}}{C}
$$

When the Earth is moving directly towards Jupiter at speed $v$ in its orbit around the Sun, based on ether theory the speed of the pulses relative to the Earth is $c+v$ and because of this increased speed, the observed period $T_{A}$ (recorded time between pulses) is shorter than $T_{o}$ since the pulses are received more quickly. The observed period is given by

$$
T_{A}=\frac{\lambda_{o}}{c+v}
$$

The measured reduction in the period $\Delta T=T_{o}-T_{A}$ is then given by

$$
\Delta T_{A}=T_{o}-T_{A}=\frac{\lambda_{o}}{c}-\frac{\lambda_{o}}{c+v}=c T_{o}\left(\frac{1}{c}-\frac{1}{c+v}\right)
$$

This reduces to

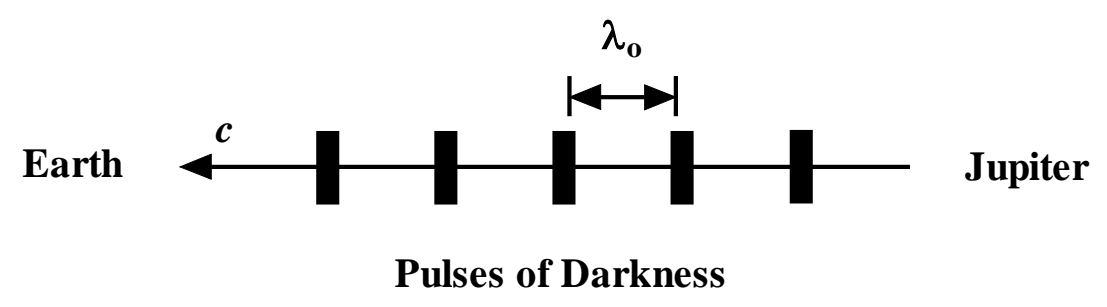

Figure 2. Pulses of darkness from Jupiter-Io system to earth. 


$$
\Delta T_{A} \simeq T_{o} \frac{v}{C}, v \ll c
$$

When the Earth is moving directly away from Jupiter, the speed of the pulses relative to the Earth is $c-v$ and because of the decreased speed, the observed period $T_{B}$ is longer than $T_{o}$ and is given by

$$
T_{B}=\frac{\lambda_{o}}{c-v}
$$

The measured increase in the period $\Delta T_{B}=T_{B}-T_{o}$ is then given by

$$
\Delta T_{B}=T_{B}-T_{o}=\frac{\lambda_{o}}{c-v}-\frac{\lambda_{o}}{c}=c T_{o}\left(\frac{1}{c-v}-\frac{1}{c}\right)
$$

This reduces to

$$
\Delta T_{B} \simeq T_{o} \frac{v}{C}, v \ll C
$$

which is identical to (17). Equations (17) and (20) can therefore be written as the ether-drift equation

$$
\Delta T=T_{o} \frac{v}{C}
$$

Equation (21) in this test predicts Io's period change $\Delta T$ while Equation (1) of the Michelson-Morley experiment predicts interferometer phase shift $\delta$ given by

$$
\delta=\frac{2 l}{\lambda} \frac{v^{2}}{c^{2}}
$$

Both Equations (21) and (1) seek to detect ether drift arising from the orbital motion of the Earth.

The measured period changes have been experimentally determined for Io and other orbiting satellites and are represented in the form of an observational relationship given by Hoyle ([17], p191): “The fractional changing of the orbital period of a satellite is simply the ratio of the speed of the Earth's motion to the speed of light." This relationship can be stated as

$$
\frac{\Delta T}{T_{o}}=\frac{v}{c}
$$

The empirical Equation (22) corresponds exactly to and therefore fully confirms the ether-drift Equation (21). Since the full dynamics of the orbits of Io and Earth are known, Io's period changes as detected on the Earth can be easily computed using classical mechanics: Consider the case of Earth moving directly away from Jupiter. During the time $T_{o}$ taken for the arrival of two successive pulses at the position of the Earth where the first pulse is received, the Earth travels a distance $v T_{o}$ beyond this point. Since $c \gg v$, the additional time $\Delta T_{B}$ needed for the second pulse to travel the additional distance $v T_{o}$ to the Earth at speed $c$ is $\Delta T_{B}=v T_{o} / c$. This result is exactly the ether-drift Equation (21) and again confirms ether drift arising from the orbital motion of the Earth. This 
first-order method involving Roemer's observation of satellite period change has succeeded where the second-order method of Michelson and Morley utilizing interferometer phase shift failed.

The observed period change for Io can be found from (22). Using Io's period $T_{o}=152850.2 \mathrm{~s}$ (1.7691 days) and the orbital speed of the Earth $v=2.978 \times 10^{4} \mathrm{~m} / \mathrm{s}$, the observed change $\Delta T$ in Io's period is given by

$$
\Delta T=T_{o} \frac{v}{C}=152850.2 \times \frac{2.978 \times 10^{4}}{2.998 \times 10^{8}}=15.2 \mathrm{~s}
$$

This means that Io's period is observed to decrease and increase by a maximum of $15.2 \mathrm{~s}$ as the Earth approaches then retreats from Jupiter in the Earth's orbit around the Sun. Using this measured change $\Delta T=15.2 \mathrm{~s}$ in the ether-drift Equation (21), we can evaluate the Earth's orbital velocity giving

$$
v=c \frac{\Delta T}{T_{o}}=2.998 \times 10^{8} \times \frac{15.2}{152,841.6}=2.98 \times 10^{4} \mathrm{~m} / \mathrm{s}
$$

The value in (24) is the measured orbital speed of the Earth and this (approximately) translational motion has been detected using Equation (21) based on Roemer's observation. It is exactly the speed that the second-order Michelson-Morley experiment [3] represented by Equation (1) failed to detect and that Stachel [11] claimed cannot be detected using first-order methods.

These observed changes in Io's period occur as a result of the changes in light speed relative to the moving Earth as the light travels through the ether. These period changes given in (17) and (20) confirm the changed light speeds $c \pm v$ relative to the Earth given in Equations (15) and (18). This can be demonstrated using actual data for Io but light speed variation can be shown more generally. In order to accomplish this, consider the case of the Earth moving towards Jupiter. Then the speed of light $c^{+}$relative to the Earth is given by

$$
c^{+}=\frac{\lambda_{o}}{T_{A}}
$$

Using (14) and (21) and noting that $T_{A}=T_{o}-\Delta T$, this becomes

$$
c^{+}=\frac{\lambda_{o}}{T_{A}}=\frac{c T_{o}}{T_{o}-\Delta T}=\frac{c T_{o}}{T_{o}-v T_{o} / c}=c\left(1-\frac{v}{c}\right)^{-1}=c\left(1+\frac{v}{c}\right), v \ll c
$$

This reduces to

$$
c^{+}=\frac{\lambda_{o}}{T_{A}}=c+v, v \ll c
$$

which is Equation (15). Similarly, for Earth receding from Jupiter, the speed of light $C^{-}$relative to the Earth is given by

$$
c^{-}=\frac{\lambda_{0}}{T_{B}}
$$

Using (14) and (21) and noting that $T_{B}=T_{o}+\Delta T$, this becomes

$$
c^{-}=\frac{\lambda_{o}}{T_{B}}=\frac{c T_{o}}{T_{o}+\Delta T}=\frac{c T_{o}}{T_{o}+v T_{o} / c}=c\left(1+\frac{v}{c}\right)^{-1}=c\left(1-\frac{v}{c}\right), v \ll c
$$


This reduces to

$$
c^{-}=\frac{\lambda_{o}}{T_{B}}=c-v, v \ll c
$$

which is Equation (18). The variable light speeds (27) and (30) relative to the Earth negate any explanation of the Roemer effect based on special relativity since in this theory, variable light speed is prohibited.

The detected ether drift is evidence of a preferred frame, which we suggest corresponds to the solar system barycentric or sun-centered inertial (SCI) frame. This is a frame that moves with the Sun but does not rotate with it and provides a convenient reference frame for a range of astronomical events. In particular, the orbital ephemerides of the planets and other bodies in the solar system are determined using a set of equations operating within this reference frame [18] [19] [20]. Based on data sets collected over a period of more than half a century of measurement, it has been determined that light travels at a constant speed $c$ in the SCI frame. Therefore, we conclude that the ether drift detected by the satisfaction of Equation (21) using changes of Io's period arising from the orbital motion of the Earth, through a medium in which light travels at speed $c$, reveals a preferred frame that corresponds to the SCI frame.

\section{Conclusions}

This paper discussed two first-order tests employing Jupiter's satellite Io for the detection of movement through the ether. These tests are Maxwell's experiment for detecting the movement of the solar system through the ether and Gift's test employing Roemer's observation for detecting the Earth's orbital motion through the ether. Maxwell's experiment requires 6 years and has never been performed while the test by Gift can be performed in a few days. The results of the latter approach yield successful detection of ether drift associated with the Earth's orbital motion and a confirmation of the existence of a preferred frame which we argue corresponds to the SCI frame. Therefore, the suggestion by Stachel [11] that first-order methods cannot detect translational motion of the Earth through the ether is shown to be false.

Also, the existence of this preferred frame violates a major tenet of relativity theory where the equivalence of all frames is demanded. Indeed, Albert Einstein in the fifth edition of his book "Relativity: The Special and the General Theory" first published in 1916 declared "But on the basis of the theory of relativity... there can be no aether-drift, nor any experiment with which to demonstrate it." The successful detection of ether drift renders this declaration also false, particularly as light speed variation relative to the orbiting Earth has been confirmed.

Thus, in view of the considerable body of theoretical, experimental and observational evidence that supports light wave transmission through an all-pervasive medium [1] [2], particularly the discovery of electromagnetic waves with speed set by measurable characteristics of that medium, this positive detection of movement through that medium (corresponding to the SCI frame) for orbital 
motion of the Earth using variations in the period of Io described in this paper, represents further confirmation of the invalidity of the relativistic worldview as argued by many [21]-[28]. It will hopefully stimulate research into the nature and properties of the luminiferous ether.

\section{Conflicts of Interest}

The author declares no conflicts of interest regarding the publication of this paper.

\section{References}

[1] Whittaker, E. (1973) A History of the Theories of Aether and Electricity: The Classical Theories. Humanities Press, New York.

[2] Schaffner, K.F. (1972) Nineteenth-Century Aether Theories. Pergamon Press, Oxford.

[3] Michelson, A.A. and Morley, E.W. (1887) On the Relative Motion of the Earth and the Luminiferous aether. American Journal of Science, S3-34, 333-345. https://doi.org/10.2475/ajs.s3-34.203.333

[4] French, A.P. (1968) Special Relativity. Nelson, London.

[5] Bailey, J. et al. (1977) Measurement of Relativistic Time Dilation for Positive and Negative Muons in a Circular Orbit. Nature, 268, 301-305. https://doi.org/10.1038/268301a0

[6] Ives, H.E. (1952) The Fitzgerald Contraction. The Scientific Proceedings of the Royal Dublin Society, 26, 9-26.

[7] Gift, S.J.G. (2010) Light Speed Invariance is a Remarkable Illusion. Physics Essays, 23, 1-5. https://doi.org/10.4006/1.3280803

[8] Levy, J. (2003) From Galileo to Lorentz... and beyond. Apeiron, Montreal.

[9] Zhang, Y.Z. (1997) Special Relativity and Its Experimental Foundations. World Scientific, Singapore.

[10] Maxwell, J.C. (1875) Ether. In: Baynes, T.S., Ed., Encyclopedia Britannica, 9th Edition, Vol. 8, Colin Macfarquhar and Andrew Bell, Edinburgh, 568-572.

[11] Stachel, J. (2005) 1905 and All That. Nature, 433, 215-217. https://doi.org/10.1038/433215a

[12] Mansouri, R. and Sexl, R.U. (1977) A Test Theory of Special Relativity: II. First-Order Tests. General Relativity and Gravitation, 8, 515-524. https://doi.org/10.1007/BF00762635

[13] Lorentz, H.A. (1953) Electromagnetic Phenomena in a System Moving with Any Velocity Less than That of Light. In: Lorentz, H.A., Einstein, A., Minkowski, H. and Weyl, H., Eds., The Principle of Relativity, Dover Publications, New York, 11-34.

[14] Saito, Y. (2005) A Discussion of Roemer's Discovery Concerning the Speed of Light. AAPPS Bulletin, 15, 9-17.

[15] Born, M. (1962) Einstein's Theory of Relativity. Dover Publications, New York.

[16] Gift, S.J.G. (2006) The Relative Motion of the Earth and the Ether Detected. Journal of Scientific Exploration, 20, 201-214.

[17] Hoyle, F. (1975) Astronomy and Cosmology: A Modern Course. Freeman, San Francisco.

[18] Aleksandrov, Y.N., Kuznetsov, B.I., Petrov, G.M. and Rzhiga, O.N. (1972) Tech- 
niques of Radar Astrometry. Soviet Astronomy, 16, 137.

[19] Ostro, S.J. (1993) Planetary Radar Astronomy. Reviews of Modern Physics, 65, 1235-1279. https://doi.org/10.1103/RevModPhys.65.1235

[20] Standish, E.M. and Williams, J.G. (2012) Orbital Ephemerides of the Sun, Moon, and Planets. In: Urban, S. and Seidelmann, P.K., Eds., The Explanatory Supplement to the Astronomical Almanac, 3rd Edition, University Science Books, California, 305-345.

[21] Nordenson, H. (1969) Relativity Time and Reality. George Allen and Unwin Ltd., London.

[22] Dingle, H. (1972) Science at the Crossroads. Martin Brian \& O’Keeffe, London.

[23] McCausland, I. (2011) A Scientific Adventure. Apeiron, Montreal.

[24] Selleri, F. (2004) Sagnac Effect: End of the Mystery. In: Rizzi, G. and Ruggiero, M.L., Eds., Relativity in Rotating Frames. Fundamental Theories of Physics, Springer, Dordrecht, 57-77. https://doi.org/10.1007/978-94-017-0528-8_6

[25] Phipps, T.E. (2016) Timekeeping Evidence Refutes the Relativity Principle. Physics Essays, 29, 62-64. https://doi.org/10.4006/0836-1398-29.1.62

[26] Engelhardt, W. (2016) Einstein's Third Postulate. Physics Essays, 29, 513-515. https://doi.org/10.4006/0836-1398-29.4.513

[27] Gift, S.J.G. (2020) Relative Simultaneity Does Not Exist. Physics Essays, 33, 515-518. https://doi.org/10.4006/0836-1398-33.4.515

[28] Gift, S.J.G. (2020) Tests of the One-Way Speed of Light Relative to a Moving Observer. Physics Essays, 33, 348-354. https://doi.org/10.4006/0836-1398-33.3.348 